\title{
Influence of different wavelengths peaks in LED units on the degree of conversion of orthodontic composites
}

\author{
Patrícia Amato ${ }^{\mathrm{a}}$, Lídia P. Martins ${ }^{\mathrm{b}}$, Alexandre Gatti $^{\mathrm{c}}$, Hermes Pretel ${ }^{\mathrm{d}}$, \\ Renato P. Martins ${ }^{\mathrm{e}, *}$ \\ ${ }^{a}$ Private Practice, Riberião Preto, Brazil \\ ${ }^{\mathrm{b}}$ Professor, Department of Orthodontics, Universidade Estadual Paulista, Araraquara, Brazil \\ ${ }^{\mathrm{C}}$ South America Representative, FlackTek Inc.-SpeedMixer, São Carlos, Brazil \\ ${ }^{\mathrm{d}}$ Adjunct Professor, Program of Restorative Dentistry, Universidade Estadual Paulista, Araraquara, Brazil \\ e Adjunct Professor, Program of Orthodontics, Universidade Estadual Paulista, and Private Practice, Araraquara, Brazil
}

\section{A R T I C L E I N F O}

\section{Article history:}

Received 16 February 2016

Received in revised form

20 September 2016

Accepted 18 October 2016

\section{Keywords:}

Composite resins

FTIR

Orthodontics

Dual-peak

\begin{abstract}
A B S T R A C T
Objective: The aim of this study was to assess the influence of adding a second wavelength peak (400 nm) to a light-emitting diode (LED) unit on the degree of conversion (DC) of two commercial orthodontic composites, as well as comparing the DC of those two composites.

Methods: Transbond XT and Opal Bond MV were the composites used for the tests, cured either with second- or third-generation LED units adjusted to produce the same power density on a given time. Analysis of DC was performed by using the Fourier-transformed infrared spectroscopy in four groups of 5 specimens each divided according to the composites and LED curing units being used. The results, which were normally distributed, were analyzed using a two-way ANOVA test.

Results: The composites showed very similar degrees of conversion when light-cured with secondgeneration (74.3\%) and third-generation (73.2\%) LED light-curing units. The Opal Bond MV composite had a higher degree of conversion (81.9\%) than the Transbond XT composite (65.7\%). No interaction was found $(P=0.56)$ between the composites and the LED curing units, regardless of their wavelength, on the degree of conversion.

Conclusion: There was no influence of either a second- or third-generation LED curing unit on orthodontic composites.
\end{abstract}

(c) 2016 World Federation of Orthodontists.

\section{Introduction}

The light-emitting diode (LED) is currently the most used light source for light-curing photosensitive materials due to its efficiency and excellent cost-benefit [1]. The first generation of LEDs emitted light at a short visible spectrum range (420 nm to $490 \mathrm{~nm}$ ) coincident with the maximum spectral absorption of camphorquinone (468 nm), which is a photo-initiator traditionally used in composites. Because of their low power, the first generation of LED units produced less energy, on a given exposure time, compared with halogen light, the light-curing unit most used until recent times [2].

All authors have completed and submitted the ICMJE Form for Disclosure of Potential Conflicts of Interest, and none were reported.

* Corresponding author: Rua Carlos Gomes, 2158, Araraquara, São Paulo, Brazil 14801340

E-mail address: dr_renatopmartins@hotmail.com (R.P. Martins).
The second generation of LED units emerged some years later, producing higher power compared with the first-generation units. Nonetheless, the short emission spectrum remained, and as a result, only composites containing camphorquinone as a photo-initiator could be effectively light-cured. This was a major drawback, because alternative photo-initiators, such as trimethylbenzoyldiphenylphosphine oxide (TPO) and phenyl-propanodione, which were developed to provide better aesthetic properties [3] and better efficiency in the light-curing reaction [4], had a different lightabsorbing spectra. To allow restorative materials to be light-cured with all photo-initiators available, high-power LED curing units having two wavelength peaks ( $400 \mathrm{~nm}$ and $465 \mathrm{~nm}$ ) gave rise to the third generation of these devices [1].

Therefore, selecting the correct light source is important to achieve the maximum degree of conversion (DC), or light-curing, possible for a particular composite. Polymerization converts the monomers present in the organic matrix of the composite into polymers [5], and this process is normally quantified by the DC, which is determined by the amount of carbon double bonds broken 
Table 1

Group distribution with the composites and light-emitting diode (LED) units used

\begin{tabular}{|c|c|c|c|c|c|}
\hline & & \multicolumn{2}{|l|}{ Composites } & \multirow{2}{*}{$\begin{array}{l}\text { Light-emission } \\
\text { peak, nm }\end{array}$} & \multirow[t]{2}{*}{ Manufacturer } \\
\hline & & Transbond XT & Opal bond MV & & \\
\hline \multirow[t]{3}{*}{ LED unit } & Ortholux & 5 & 5 & 463.87 & 3M/Unitek, Monrovia, CA \\
\hline & Valo Cordless & 5 & 5 & 459.11 & Ultradent Products Inc, South Jordan, UT \\
\hline & & & & 403.97 & \\
\hline \multicolumn{2}{|c|}{ Batch number } & N182301 & $\mathrm{C} 013$ & & \\
\hline \multicolumn{2}{|c|}{ Manufacturer } & 3M/Unitek, Monrovia, CA & Opal Orthodontics, South Jordan, UT & & \\
\hline
\end{tabular}

during the polymerization process [6]. As the DC in a composite gets higher, the physical-chemical properties are improved [7-13], such as greater hardness [10-13], lower solubility and degradation over time [7], smaller rate of residual monomer, which has been related to adverse biological effects $[8,9,11,12]$, and possibly increased shear bond strength of orthodontic brackets [14]. Because it has been established that the use of third-generation LED light-curing units improves the DC of composites containing TPO, and that the presence of TPO may not be indicated by the manufacturer [13], it would be a good idea to use these devices whenever possible. However, there is controversy in the literature about whether thirdgeneration LED curing units could compromise the light-curing process of composites containing camphorquinone only as photoinitiators $[13,15]$. Even though there are studies comparing the use of second- and third-generation LED light-curing units in restorative composites, the optimal energy density has not yet been determined for these devices in terms of effect of light-emission peaks, and most importantly, there is only one report on orthodontic composites being cured with these lights [16].

There are several photo-activated orthodontic composites commercially available, all having different chemical compositions in which the photo-initiator is not always revealed by the manufacturer. For this reason, the light-curing kinetics of a composite do not necessarily apply to another. Therefore, the objective of this study was to assess the influence of second- and third-generation LED light-curing units, operating at the same energy level, on the DC of two orthodontic composites.

\section{Materials and methods}

Twenty composite discs of 6-mm diameter and 1-mm thickness were made and divided into two groups according to their manufacturer: group 1 disks were produced with Transbond XT (3M Unitek, Monrovia, CA) and group 2 disks were produced with Opal Bond MV (Ultradent Products Inc, South Jordan, UT) (Table 1).

A metallic mold was placed over a 10 -mm-thick glass slide and the composite was inserted to it in one increment, and both upper and lower surfaces were covered with a polyester band. A 1-mm-thick glass slide was placed over the polyester tape, on the disc's upper surface, before photo-activation, so that the incidence direction of the light beam was perpendicular to the sample, allowing the distance from the light source and the composite to be standardized.

Each group was divided into two subgroups containing five composite discs each, which were light-cured with a different LED light-curing unit. In one subgroup, a second-generation LED lightcuring unit (Ortholux; 3M Unitek) was used, whereas in the other subgroup, a third-generation LED light-curing unit (Valo Cordless; Ultradent Products Inc) was used (Table 2).

Times and power were standardized in such a way that both devices could yield the same energy density to the composite discs, differing only on the wavelength emitted. When the secondgeneration LED was used, exposure time was 20 seconds at a power density of $860 \mathrm{~mW} / \mathrm{cm}^{2}$, with the light source tip in contact with the glass slide. The same exposure time was used for the third-generation LED unit, but the device's light source tip was kept $17 \mathrm{~mm}$ away from the glass slide through a supporting apparatus especially made for this article, allowing both light sources to yield the same power density.

After curing, the composite discs were individually stored in dark containers until the moment they were prepared for analysis of the DC using the Fourier-transformed infrared (FTIR) spectroscopy technique.

Five light-cured composite tablets were made for each subgroup using a respective composite disc. Each tablet had $8 \mathrm{~mm}$ of diameter and was prepared with a specific apparatus (PerkinElmer, Beaconsfield Bucks, England), being compressed under 10 tons for 60 seconds. Each tablet contained $5 \mathrm{mg}$ of composite, which was ground to powder from each of the cured discs, and mixed with $100 \mathrm{mg}$ potassium bromate. For DC analysis, these tablets were compared with other five tablets made exactly the same from the respective composite, but not light-cured.

The light-cured and non-light-cured tablets from each subgroup were analyzed by a spectrophotometer (Spectrum 2000; Perkin-Elmer) for spectral acquisition. All measurements were performed with the device operating at absorbance mode, with an interval between 4000 and $400 \mathrm{~cm}^{-1}$, 32 scans, and resolution of $4 \mathrm{~cm}^{-1}$. Spectral analysis was conducted by using the equipment's dedicated software (Spectrum v 5.3.1 for Windows; Perkin-Elmer).

The DC in percentage was calculated by analyzing the spectra obtained through the decrease in the band intensity of vinyl methacrylate group double bonds, whose energy absorption is in the region of $1637 \mathrm{~cm}^{-1}$ compared with the band intensity of aromatic double bonds, which is in the region of $1610 \mathrm{~cm}^{-1}$. The latter was used as an inner sample pattern, as it does not change after a polymerization reaction.

The SPSS software (v.16,0; SPSS, Chicago, IL) was used for statistical analysis. Because the values were found to be normally distributed, a two-way ANOVA $(P=0.05)$ was used to assess the influence of composite and LED unit on the DC, as well as to find a possible interaction between those two factors.

\section{Results}

There was no difference $(P=0.60)$ between the DCs when second-generation and third-generation LED light-curing units were used (Tables 3 and 4).

Table 2

Descriptive data of the factors analyzed by the two-way ANOVA

\begin{tabular}{llll}
\hline & \multicolumn{2}{l}{ Composites } & Total \\
\cline { 2 - 3 } & Group 1 & Group 2 & \\
\hline LED unit & $65.8(8.0)$ & $80.7(3.8)$ & $73.2(9.8)$ \\
2nd Generation & $65.6(2.2)$ & $83.0(1.2)$ & $74.3(9.3)$ \\
3rd Generation & $65.7(5.5)$ & $81.9(2.9)$ & \\
Total &
\end{tabular}

Means and SDs of the degree of conversion are shown for the wavelength (second and third generations) and for the composites (group 1 and group 2). 
Table 3

Two-way ANOVA for degree of conversion

\begin{tabular}{llllcc}
\hline Factors & $\begin{array}{l}\text { Type III sum } \\
\text { of Squares }\end{array}$ & $d f$ & $\begin{array}{l}\text { Mean } \\
\text { Square }\end{array}$ & F & $P$ \\
\hline LED & 6.25 & 1 & 6.252 & 0.293 & 0.60 \\
Composite & 1306 & 1 & 1306 & 61.28 & $<0.001^{*}$ \\
Composite $\times$ LED unit & 7.7 & 1 & 7.705 & 0.362 & 0.56 \\
\hline
\end{tabular}

LED, light-emitting diode.

* Significant difference.

On the other hand, a difference was found $(P<0.001)$ between the DCs of the composites tested (Table 3). Group 1 showed the lowest DC (65.7\%) compared with group 2 (81.9\%) (Table 5).

No interaction was found $(P=0.56)$ between the composites and the LED curing units, regardless of their wavelength, on the DC (Table 3).

\section{Discussion}

Adding a light-emission peak at $400 \mathrm{~nm}$ but maintaining the same energy level did not increase the DC of the composites compared with a single peak ranging from $430 \mathrm{~nm}$ to $520 \mathrm{~nm}$ for the experimental light-curing conditions. This addition was expected to increase the DC of the composites if any of the composites contained an alternative photo-initiator, such as TPO $[3,4,13]$ or phenyl-propanodione $[4]$ in their composition. However, because of the low power of the comparisons (8\%), we could not securely rule out the absence of such photo-initiators in one these composites. In the literature, there are five studies comparing the DCs of different composites light-cured with second- and thirdgeneration LED light-curing units [13,15-18]. Despite their controversial results, none of these articles standardized the energy density of the LEDs, isolating the effect of an additional lightemission peak, as we have done in this article.

The standardized energy density used in this study was very important because it is directly proportional to the DC $[19,20]$. If both devices used showed different energy-density levels, this factor might have influenced the comparison of the DC between the composites tested, confounding the results. Moreover, different levels of energy density might have also influenced the results in terms of comparison between second- and third-generation LED light-curing units.

The DCs of the composites tested were different, as expected, because they are related to the chemical composition of the composites [21-27]. The infrared spectra analysis (Fig. 1) clearly demonstrates that their compositions are distinct, because the spectrum of each one has different absorption profiles. Monomer concentration, chemical structure [24,25], and viscosity [23,26], as well the amount [22,27] and type [13] of photo-initiators existing in the organic matrix will all have a direct effect on the polymerization kinetics.

Given the same energy level, Opal Bond MV and Transbond XT composites had DCs of $81.9 \%$ and $65.7 \%$, respectively. Even though the latter showed a lower conversion, it was still between $55 \%$ and $75 \%$, which is the range of conversion usually considered adequate under conventional irradiation (i.e., halogen light for 40 seconds) $[28,29]$. Two important factors need to be mentioned when DC is analyzed in orthodontic composites: first is that a positive

Table 4

Means and SDs for the values of DC for the LED light-curing units used (second and third generations)

\begin{tabular}{lll}
\hline LED unit & DC, \% & SD \\
\hline 2nd generation & 73.2 & 8.01 \\
3rd generation & 74.3 & 2.23 \\
\hline
\end{tabular}

DC, degree of conversion; LED, light-emitting diode.
Table 5

Means and SDs for the values of DC for the composites used

\begin{tabular}{lll}
\hline Composites & DC, \% & SD \\
\hline G1 & 65.7 & 3.82 \\
G2 & 81.9 & 1.22 \\
\hline
\end{tabular}

DC, degree of conversion; G, group.

correlation exists between DC and shear bond strength of bonded brackets [14], and the other is that a low DC might favor the release of bisphenol-A, a chemical that exhibits estrogenicity [30] and is toxic $[11,12]$. Thus, it appears that an immediate high DC after curing is a desirable characteristic of an orthodontic composite.

Of the two orthodontic composites analyzed, although Transbond XT has been extensively evaluated by the literature on DC [14,21,31-35], showing values ranging from $39 \%$ to $83 \%$, the DC of Opal Bond has been evaluated by only one report [16]. Because of differences in the methodologies and light-curing protocols used by all those articles, which were different from ours, as well, it is not possible to compare the results directly. Three of these studies used halogen light or plasma arc, which emit a different light spectrum [31,34,35], or did not report the total energy applied to the composites [14,21,31,35]. Three studies used second-generation LED light-curing units [21,32,33], but one [33] used a light-curing method and analysis of conversion different from ours, whereas the other two [21,32] used different activation times, power levels, and total energies. The only report that analyzed DC on Opal Bond MV16 showed a lower result than we did (61\%), but used a completely different methodology than we did.

From the clinical point of view, the findings reported here are important. First, one can conclude that choosing the composite is more important than adding a light-emission peak, according to our test conditions, and, second, by choosing the correct composite, the orthodontist can achieve better physical-chemical properties compared with other composites, given the same light-curing time and same energy level.

\section{Conclusion}

With regard to the DC of the two orthodontic composites evaluated, namely, Opal Bond MV and Transbond XT, both lightcured with second- and third-generation LED light-curing units operating at the same energy level, one can conclude the following:

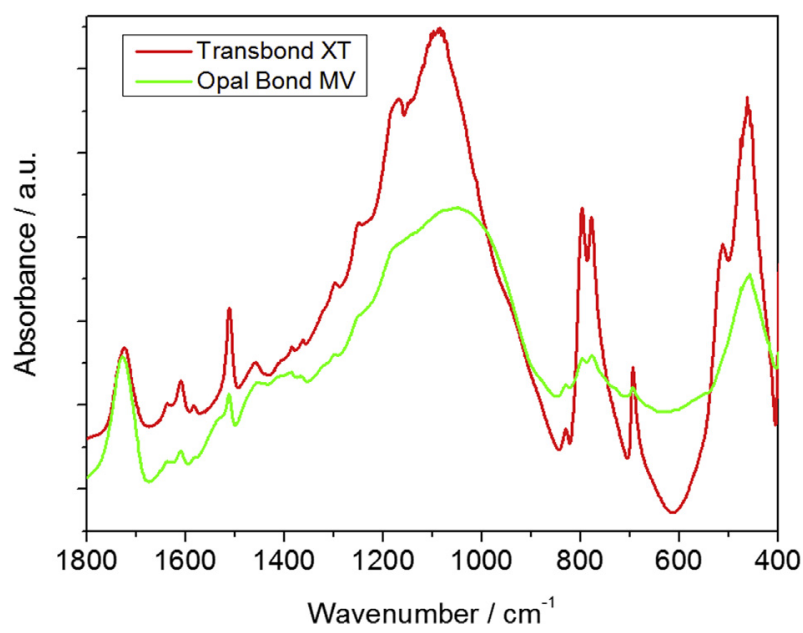

Fig. 1. Infrared spectrum of the different composites tested. The difference in chemical compositions among the different trademarks and manufactures can be observed in the fingerprint region $\left(1500 \mathrm{~cm}^{-1}\right.$ and $\left.400 \mathrm{~cm}^{-1}\right)$ of the infrared spectra of the nonpolymerized adhesives (a.u. = absorbance units). 
(1) type of LED light-curing unit had no influence on the DC of the orthodontic composites, and (2) there was a difference in the DC of the composites, with Opal Bond MV presenting values higher than those of Transbond XT.

\section{References}

[1] Rueggeberg FA. State-of-the-art: dental photocuring-a review. Dent Mater 2011;27:39-52.

[2] Dunn WJ, Bush AC. A comparison of polymerization by light-emitting diode and halogen-based light-curing units. J Am Dent Assoc 2002;133:335-41.

[3] Arikawa H, Takahashi H, Kanie T, Ban S. Effect of various visible light photoinitiators on the polymerization and color of light-activated resins. Dent Mater J 2009;28:454-60.

[4] Park YJ, Chae KH, Rawls HR. Development of a new photoinitiation system for dental light-cure composite resins. Dent Mater 1999;15:120-7.

[5] Peutzfeldt A. Resin composites in dentistry: the monomer systems. Eur J Oral Sci 1997; 105:97-116.

[6] Decker C. Kinetic analysis and performance of UV-curable coatings. In: Pappas SP, editor. Radiation curing, science and technology. New York: Plenum Press; 1992. p. 135-79.

[7] Söderholm KJ, Zigan M, Ragan M, Fischlschweiger W, Bergman M. Hydrolytic degradation of dental composites. J Dent Res 1984;63:1248-54.

[8] Rathbun MA, Craig RG, Hanks CT, Filisko FE. Cytotoxicity of a BIS-GMA dental composite before and after leaching in organic solvents. J Biomed Mater Res 1991;25:443-57.

[9] Geurtsen W, Lehmann F, Spahl W, Leyhausen G. Cytotoxicity of 35 dental resin composite monomers/additives in permanent 3T3 and three human primary fibroblast cultures. J Biomed Mater Res 1998;41:474-80.

[10] Bouschlicher MR, Rueggeberg FA, Wilson BM. Correlation of bottom-to-top surface microhardness and conversion ratios for a variety of resin composite compositions. Oper Dent 2004;29:698-704.

[11] Ahrari F, Tavakkol Afshari J, Poosti M, Brook A. Cytotoxicity of orthodontic bonding adhesive resins on human oral fibroblasts. Eur J Orthod 2010;32:688-92.

[12] Malkoc S, Corekci B, Ulker HE, Yalçin M, Sengün A. Cytotoxic effects of orthodontic composites. Angle Orthod 2010;80:571-6.

[13] Santini A, Miletic V, Swift MD, Bradley M. Degree of conversion and microhardness of TPO-containing resin-based composites cured by polywave and monowave LED units. J Dent 2012;40:577-84.

[14] Chidipothu HR, Chandrasekhar S. Evaluation of degree of cure and shear bond strengh of a color changing light cure adhesive. J Indian Orthod Soc 2012;46:334-9.

[15] Miletic V, Santini A. Micro-Raman spectroscopic analysis of the degree of conversion of composite resins containing different initiators cured by polywave or monowave LED units. J Dent 2012;40:106-13.

[16] Santini A, McGuinness N, Nor NA. Degree of conversion of resin-based orthodontic bonding materials cured with single-wave or dual-wave LED light-curing units. J Orthod 2014;41:292-8.

[17] Aguiar FH, Georgetto MH, Soares GP, et al. Effect of different light-curing modes on degree of conversion, staining susceptibility and stain's retention using different beverages in a nanofilled composite resin. J Esthet Restor Dent 2011;23:106-14.

[18] Ribeiro BC, Boaventura JM, Brito-Gonçalves J, Rastelli AN, Bagnato VS, Saad JR. Degree of conversion of nanofilled and microhybrid composite resins photo-activated by different generations of LEDs. J Appl Oral Sci 2012;20:212-7.

[19] Halvorson RH, Erickson RL, Davidson CL. Energy dependent polymerization of resin-based composite. Dent Mater 2002;18:463-9.

[20] Peutzfeldt A, Asmussen E. Resin composite properties and energy density of light cure. J Dent Res 2005;84:659-62.

[21] Corekci B, Malkoc S, Ozturk B, Gunduz B, Toy E. Polymerization capacity of orthodontic composites analyzed by Fourier transform infrared spectroscopy. Am J Orthod Dentofacial Orthop 2011;139:E299-304.

[22] Leprince JG, Hadis M, Shortall AC, et al. Photoinitiator type and applicability of exposure reciprocity law in filled and unfilled photoactive resins. Dent Mater 2011;27:157-64.

[23] Lovell LG, Newman SM, Bowman CN. The effects of light intensity, temperature, and comonomer composition on the polymerization behavior of dimethacrylate dental resins. J Dent Res 1999;78:1469-76.

[24] Gonçalves F, Kawano Y, Pfeifer C, Stansbury JW, Braga RR. Influence of BisGMA, TEGDMA, and BisEMA contents on viscosity, conversion, and flexural strength of experimental resins and composites. Eur J Oral Sci 2009; $117: 442-6$

[25] Asmussen E, Peutzfeldt A. Influence of composition on rate of polymerization contraction of light-curing resin composites. Acta Odontol Scand 2002;60:146-50.

[26] Sideridou I, Tserki V, Papanastasiou G. Effect of chemical structure on degree of conversion in light-cured dimethacrylate-based dental resins. Biomaterials 2002;23:1819-29.

[27] Musanje L, Ferracane JL, Sakaguchi RL. Determination of the optimal photoinitiator concentration in dental composites based on essential material properties. Dent Mater 2009;25:994-1000.

[28] Ferracane JL, Greener EH. The effect of resin formulation on the degree of conversion and mechanical properties of dental restorative resins. J Biomed Mater Res 1986;20:121-31.

[29] Yoon TH, Lee YK, Lim BS, Kim CW. Degree of polymerization of resin composites by different light sources. J Oral Rehabil 2002;29:1165-73.

[30] Olea N, Pulgar R, Perez P, et al. Estrogenicity of resin-based composites and sealants used in dentistry. Environ Health Perspect 1996;104:298-305.

[31] Eliades T, Eliades G, Brantley WA, Johnston WM. Polymerization efficiency of chemically cured and visible light-cured orthodontic adhesives: degree of cure. Am J Orthod Dentofacial Orthop 1995;108:294-301.

[32] Cerveira GP, Berthold TB, Souto AA, Spohr AM, Marchioro EM. Degree of conversion and hardness of an orthodontic resin cured with a light-emitting diode and a quartz-tungsten-halogen light. Eur J Orthod 2010;32:83-6.

[33] Carvalho Fde A, Almeida RC, Almeida MA, Cevidanes LH, Leite MC. Efficiency of light-emitting diode and halogen units in reducing residual monomers. Am J Orthod Dentofacial Orthop 2010;138:617-22.

[34] Bang HC, Lim BS, Yoon TH, Lee YK, Kim CW. Effect of plasma arc curing on polymerization shrinkage of orthodontic adhesive resins. J Oral Rehabil 2004;31:803-10.

[35] Jagdish N, Padmanabhan S, Chitharanjan AB, et al. Cytotoxicity and degree of conversion of orthodontic adhesives. Angle Orthod 2009;79:1133-8. 